\title{
Physical mechanisms involved in grooved flat heat pipes: experimental and numerical analyses
}

\author{
Stéphane Lips, Frédéric Lefèvre*, Jocelyn Bonjour \\ Université de Lyon, CNRS \\ INSA-Lyon, CETHIL, UMR5008, F-69621, Villeurbanne, France \\ Université Lyon 1, F-69622, France \\ *(corresponding author: frederic.lefevre@insa-lyon.fr)
}

\begin{abstract}
An experimental database, obtained with flat plate heat pipes (FPHP) with longitudinal grooves is presented. The capillary pressure measured by confocal microscopy and the temperature field in the wall are presented in various experimental conditions (vapour space thickness, filing ratio, heat transfer rate, tilt angle, fluid). Coupled hydrodynamic and thermal models are developed. Experimental results are compared to results of numerical models. Physical mechanisms involved in grooved heat pipes are discussed, including the boiling limit and the effect of the interfacial shear stress. Finally, recommendations for future experimental and theoretical research to increase the knowledge on FPHP are discussed.
\end{abstract}

Keywords: flat plate heat pipe; capillary pressure; condensation; evaporation; interfacial shear stress; boiling limit

\begin{tabular}{|c|c|c|c|}
\hline \multicolumn{4}{|c|}{ Nomenclature } \\
\hline$a$ & accommodation coefficient & Subscri & \\
\hline$A$ & cross section, $\mathrm{m}^{2}$ & cap & capillary \\
\hline$c_{\min }$ & minimum aspect ratio & cond & condensation \\
\hline$f$ & friction coefficient & evap & evaporation \\
\hline$f_{r}$ & filling ratio & $\mathrm{f}$ & fin \\
\hline$g$ & gravitational acceleration, $\mathrm{m} \cdot \mathrm{s}^{-2}$ & $\mathrm{~g}$ & groove \\
\hline$h_{\mathrm{lv}}$ & latent heat of vaporization, $\mathrm{J} \mathrm{kg}^{-1}$ & $\mathrm{i}$ & node number \\
\hline$h$ & height, $\mathrm{m}$ & int & interface \\
\hline$l$ & width, $\mathrm{m}$ & 1 & liquid \\
\hline$L$ & length, m & long & longitudinal \\
\hline$m$ & mass, kg & $\max$ & maximum \\
\hline $\bar{M}$ & mass per mole, $\mathrm{kg} \cdot \mathrm{mol}^{-1}$ & sat & saturation \\
\hline$n$ & number of nodes & $\operatorname{trans}$ & transversal \\
\hline$N$ & number of grooves & $\mathrm{v}$ & vapour \\
\hline$P$ & pressure, $\mathrm{Pa}$ & $\mathrm{W}$ & wall \\
\hline Po & Poiseuille number & & \\
\hline$q$ & heat flux, W.m ${ }^{-2}$ & Greeks & ymbols \\
\hline$Q$ & heat transfer rate, $\mathrm{W}$ & $\theta$ & slope of the FPHP, rad \\
\hline$\vec{r}$ & radius of curvature, $\mathrm{m}$ & $\rho$ & density, kg. $\mathrm{m}^{-3}$ \\
\hline$r_{0}$ & radius of curvature in the condenser, $\mathrm{m}$ & $\sigma$ & surface tension, N.m ${ }^{-1}$ \\
\hline $\bar{R}$ & ideal gas constant, J.K $\mathrm{K}^{-1} \cdot \mathrm{mol}^{-1}$ & $\tau$ & shear stress, N.m ${ }^{-2}$ \\
\hline $\operatorname{Re}$ & Reynolds number & $\delta$ & liquid film thickness, $\mathrm{m}$ \\
\hline$r_{t h}$ & thermal resistance, $\mathrm{K} \mathrm{W}^{-1}$ & & \\
\hline$R_{t h}$ & thermal resistance per unit area, $\mathrm{K} \cdot \mathrm{m}^{2} \cdot \mathrm{W}^{-1}$ & & \\
\hline$S$ & surface area, $\mathrm{m}^{2}$ & & \\
\hline$T$ & temperature, $\mathrm{K}$ & & \\
\hline$u$ & velocity, $\mathrm{m} . \mathrm{s}^{-1}$ & & \\
\hline$x, y, z$ & coordinates, $\mathrm{m}$ & & \\
\hline
\end{tabular}




\section{Introduction}

Capillary heat pipes and two-phase thermosyphons are well-known heat transfer devices, having numerous industrial applications (electronic cooling, aeronautics, space ...). Nevertheless, their integration in electronic or microelectronic systems implies their miniaturisation and an increase of the heat fluxes to be transferred. To face these new challenges, original two-phase cooling devices were developed, such as micro heat pipes or flat plate heat pipes (FPHP) [1]. A FPHP is a hermetic cavity of small thickness partially filled with a two-phase working fluid. Heat sources and heat sinks are located anywhere on the walls of the cavity, with the other parts being insulated. Vapour is generated at the heat source (evaporator) and it condenses at the heat sink (condenser). The liquid returns from the evaporator to the condenser through a capillary structure that can be made of micro-grooves, meshes or sintered powder wicks.

Since the nineties, several works were published on this type of systems and a lot of numerical or analytical models were proposed in order to predict their thermal performance and/or their capillary limit. The aim of the models is to calculate liquid and vapour pressures and velocities and the temperature field in the wall from heat sources to heat sinks. For grooved capillary structure, hydrodynamic models are based on the balance equations and the Young-Laplace law, which connects the liquid and vapour pressures to meniscus curvature radii in the grooves [2-9]. For other capillary structures (mainly meshes, sintered powder wicks or crossed grooves), such an approach is not possible. The flow in the capillary structure is modelled by the Darcy's law in 2D [10] or 3D [11,12].

In thermal models, longitudinal heat conduction in the wall and transversal heat transfer from the vapour to the wall have to be taken into account. Thermal and hydrodynamic models are highly coupled since the mass balance depends on the heat conduction in the wall. Furthermore, the transversal heat flux depends on the shape of the liquid in the capillary structure. As an example, for grooved capillary structures, the transversal heat transfer depends on the meniscus curvature radius in the grooves that is calculated by hydrodynamic models [13-15].

Although theoretical works on FPHP are based on physical descriptions including both thermal and hydrodynamic parameters, most of the experimental works provide only external temperature measurements to characterize the systems [1]. Furthermore, the studied FPHP are always opaque, which prevents observations inside the system in working conditions. This is for example the case in one of the most comprehensive study on flat plate heat pipes [16].

To cope with this lack of experimental information, we developed several experimental benches dedicated to the observation of the liquid-vapour interface inside FPHP [4,17-19]. FPHP were realised by sealing a transparent plate on the capillary structure to allow confocal microscopy measurements. In [4], a FPHP with longitudinal grooves was tested for the cooling of a fuel cell. The system under investigation was made of copper and filled with methanol. Because of its specific application, it had a 
wide evaporating area $\left(190 \times 90 \mathrm{~mm}^{2}\right)$ compared to the condenser area $\left(30 \times 90 \mathrm{~mm}^{2}\right)$. As a result, the capillary limit was observed for a rather small heat flux $\left(0.9 \mathrm{Wcm}^{-2}\right)$. Temperature measurements on the wall and meniscus curvature radius measurements in the grooves were compared to a model of the FPHP [13]. Both the coupled thermal and hydrodynamic models were in good agreement with the experimental results. In [19], a radial FPHP made of silicon was tested. The confocal method allowed measuring both the liquid film shape inside the radial grooves and the condensate films on the fins, which was not assessable with copper. Liquid film thicknesses lower than $10 \mu \mathrm{m}$ and down to $2 \mu \mathrm{m}$ were measured. Measurements showed that the condensate film forms a drop whose curvature is opposed to that of the meniscus in the grooves.

In [17] and [18] another FPHP in copper with longitudinal grooves was tested, but with dimensions $\left(70 \times 90 \mathrm{~mm}^{2}\right)$ closer to those typically encountered in electronic cooling. Therefore, higher heat fluxes were applied to the system before the dry-out of the evaporator. In [17], nucleate boiling was observed at the evaporator for relative small heat fluxes $\left(\sim 3 \mathrm{~W} / \mathrm{cm}^{2}\right)$. The presence of nucleate boiling improves the thermal performance of the flat heat pipe, and it was emphasized that boiling does not constitute an operation limit, contrary to what is generally stated in the literature. The dryout of the evaporator was observed for heat fluxes much higher than the heat flux of onset of nucleate boiling. Nevertheless, it was not possible to testify if the dryout was due to a capillary limit or if it was inherent to the boiling phenomenon itself. In [18], meniscus curvature radius measurements and temperature measurements were presented for different vapour space thicknesses and various filling ratios. Experimental results showed that the liquid distribution in the FPHP - and thus its thermal performance - depends strongly on both the filling ratio and the vapour space thickness. Theoretical investigations were presented to model the distribution of the liquid inside the FPHP in function of the filling ratio and the vapour space thickness.

In this paper, an experimental database obtained with the latter-mentioned FPHP and a comparison with a numerical model are presented. This database includes results already presented in previous papers $[4,17,18]$, but also new results obtained in different experimental conditions. The paper mostly comprises three parts. The first part describes the numerical model with its assumptions. The second part is dedicated to the presentation of the experimental bench and some experimental results. In the last part, some key features on FPHP modelling are discussed by comparing the model and the database.

\section{Modelling of a FPHP with longitudinal grooves}

In this section, a 1D hydrodynamic model coupled to a thermal model is presented for a FPHP with longitudinal grooves. The hydrodynamic model is similar to previous works on micro heat pipes and flat plate heat pipes [2-9]. The thermal model is a simplified version of the thermal model presented by Lefèvre et al. [13]. Evaporator and condenser models are not linked to the shape of the 
liquid-vapour interface but heat transfer phenomenon are considered as constant in the evaporator and in the condenser.

\subsection{Hydrodynamic modelling}

The FPHP is divided into several control volumes, for which the balance equations are written for both the liquid and the vapour. As the FPHP is symmetrical, the control volume contains one groove and half of a fin on each side of the groove. The coupled mass and energy balance equations are written as:

$$
\begin{aligned}
& \frac{d\left(A_{l} u_{l}\right)}{d x} d x=-\frac{q l_{f+g}}{\rho_{l} h_{l v}} d x \\
& \frac{d\left(A_{v} u_{v}\right)}{d x} d x=\frac{q l_{f+g}}{\rho_{v} h_{l v}} d x
\end{aligned}
$$

where $x$ is the coordinate along the axis, $A$ is the cross section, $u$ the velocity, $q$ the rate of phase change calculated in the thermal model, $l_{\mathrm{f}+\mathrm{g}}$ the width of the control volume (which includes one fin and one groove), $\rho$ the density and $h_{\mathrm{lv}}$ the latent heat of vaporization of the fluid. Subscripts 1 and $\mathrm{v}$ denote respectively the liquid and the vapour.

In the literature, different expressions can be found for the momentum balance equations. Equation (3) presents a general mathematical formulation for the liquid including all the physical terms that were found in the literature:

$\rho_{l} \frac{d\left(A_{l} u_{l}^{2}\right)}{d x} d x=-A_{l} \frac{d P_{l}}{d x} d x-P_{l} \frac{d A_{l}}{d x} d x+P_{v} \frac{d A_{l}}{d x} d x$

$$
+\frac{\sigma}{r} \frac{d A_{l}}{d x} d x-\rho_{l} A_{l} g \sin \theta d x+\left|\tau_{l w}\right| L_{l w} d x+\left|\tau_{\text {int }}\right| L_{\text {int }} d x+\frac{d m_{l}}{d x}\left(u_{l}-u_{\text {int }}\right) d x
$$

$P$ is the pressure, $\sigma$ the surface tension, $r$ the curvature radius of the liquid-vapour interface, $L$ the wetted length, $g$ the gravitational acceleration, $\theta$ the inclination of the FPHP, $\tau$ the shear stress and $m_{1}$ the mass of liquid that vaporizes or condenses in the control volume. Subscripts w and int denote the wall and the liquid-vapour interface respectively. The terms of this equation are:

- (1) the momentum balance in the control volume,

- (2) and (3) liquid pressure forces,

- (4) vapour pressure forces,

- (5) capillary forces, 
- (6) gravity forces,

- (7) fluid-wall shear stress,

- (8) interfacial shear stress,

- (9) transfer of momentum due to phase change.

Other terms can be included in specific applications. For example, forces due to electric field have to be included for electro-hydrodynamically (EHD) driven heat pipes.

Table 1 summarises the terms that are taken into account in different publications. This table shows that the mathematical formulation of the momentum balance equation is not established well and the assumptions are not always written in the papers. Terms due to pressure forces (2 to 5 ) are often roughly described. On each side of the volume control (figure 1), both the pressure and the liquid cross-section vary. As the meniscus curvature radius decreases from the condenser to the evaporator, the liquid cross section decreases too. However, in most of the papers, the liquid cross-section variation is not taken into account in the momentum equations and thus, only term (2) appears. Some authors have taken into account this variation by introducing term (3). However, this term is mostly counterbalanced by the term (4): the part of the cross section that is no longer subject to the liquid pressure is subject to the vapour pressure. If the groove width is large enough, which was the case in [4] $(\lg =400 \mu \mathrm{m})$, the sum of terms (3) and (4) is negligible compared to term (2). However, this mathematical formulation is not correct too. This is highlighted for a FPHP with radial grooves, whose width decreases from the periphery to the centre of the FPHP [19]. In such grooves, even without heat dissipation at the evaporator, the liquid-cross section varies along the grooves but there is obviously no pressure variation in the grooves (as there is no fluid motion). This is due to capillary forces (term 5), that act on the liquid-vapour interface and counterbalance the non equilibrium due to liquid and vapour pressure forces. Finally, the variation of the liquid cross-section does not generate a force on the control volume since it compensated by the capillary forces. As a result, only term (2) has to appear in the momentum balance equation, which is the case in most of the paper in the literature but the derivation of this equation is not trivial and has never clearly been explained.

Term (9), which represents the transfer of momentum due to evaporation or condensation is taken into account in fundamental studies [20,21], but not in publications dedicated to heat pipes. This term is introduced by Longtin et al. [2] and assumed to be negligible. In our experimental conditions, we verified that this term was always negligible too.Evaporation or condensation flow rates are small enough to neglect this acceleration force.

Note that Suman et al. $[7,8]$ reduced the momentum balance equation to its minimal formulation by removing all the terms that were negligible in their configuration. As a result, they can solve the equation analytically. 
By taking into account the previous assumptions, the momentum balance equations for the liquid and the vapour phase can be written as:

$$
\begin{aligned}
& \rho_{l} \frac{d\left(A_{l} u_{l}^{2}\right)}{d x} d x=-A_{l} \frac{d P_{l}}{d x} d x-\rho_{l} A_{l} g \sin \theta d x+\left|\tau_{l w}\right| L_{l w} d x+\left|\tau_{\text {int }}\right| L_{\text {int }} d x \\
& \rho_{v} \frac{d\left(A_{v} u_{v}^{2}\right)}{d x} d x=-A_{v} \frac{d P_{v}}{d x} d x-\left|\tau_{v w}\right| L_{v w} d x-\left|\tau_{\text {int }}\right| L_{\text {int }} d x
\end{aligned}
$$

In these equations, shear stress calculations are the most important feature. The flow is assumed to be laminar for both the liquid and the vapour, which was always verified during our experiments from the calculation of the Reynolds number. For laminar flows, the liquid-wall and vapour-wall shear stresses can be calculated as:

$$
\tau_{w}=\frac{1}{2} \rho u^{2} f \quad \text { with } f=\frac{P o}{R e}
$$

where $f$ is the friction factor, $P o$ the Poiseuille number and Re the Reynolds Number. The Poiseuille number depends on the geometry of the channel.

As the liquid velocity is very low compared to the vapour velocity, the vapour Poiseuille number is calculated considering a single rectangular channel of thickness $h_{\mathrm{v}}$ and width $N \times l_{f+\mathrm{g}}$, where $N$ is the number of grooves. As a result, the interfacial shear stress is equal to the vapour-wall shear stress. For the liquid flow, the liquid-wall shear stress is calculated considering a free liquid-vapour interface. The liquid Poiseuille number is determined by considering a rectangular channel of height $2 h_{\mathrm{g}}$ (where $h_{\mathrm{g}}$ is the height of the groove) and width $l_{\mathrm{g}}$. The interfacial shear stress for the liquid is equal to that calculated for the vapour.

For a rectangular channel, the Poiseuille number can be calculated with the Shah and London correlation [22]:

$$
f R e=24\left(1-1.3553 c_{\text {min }}+1.9467 c_{\text {min }}^{2}-1.7012 c_{\text {min }}^{3}+0.9564 c_{\text {min }}^{4}-0.2537 c_{\text {min }}^{5}\right)
$$

where $c_{\min }$ is the minimal aspect ratio of the rectangular channel. It has to be noted that the circular shape of the liquid in a rectangular channel was modelled using a CFD software for comparison. For a square groove with a width equal to $400 \mu \mathrm{m}$, the difference is lower than $5 \%$ for meniscus radii higher than $500 \mu \mathrm{m}$, which validates the use of correlation (7) in this work.

In equations (1), (2), (4), and (5), the cross sections and the wetted lengths depend on the liquid vapour interface shape, and especially on the meniscus curvature radius in the groove $r$. The derivative of the Young-Laplace law with respect to $x$ is the last equation of the model. It links the liquid and vapour pressures to the meniscus curvature radius: 


$$
\frac{d P_{l}}{d x}=\frac{d P_{v}}{d x}-\frac{d}{d x}\left(\frac{\sigma}{r}\right)
$$

These five first-order differential equations (1), (2), (4), (5) and (8) form a set of non-linear coupled equations that are solved by a fourth-order Runge-Kutta method using the following boundary conditions:

$$
\begin{array}{ll}
\left.u_{l}\right|_{x=L_{g}}=\left.u_{v}\right|_{x=L_{g}}=0 & ;\left.P_{v}\right|_{x=L_{g}}=P_{\text {sat }} \\
\left.r\right|_{x=L_{g}}=r_{\max } & ;\left.P_{l}\right|_{x=L_{g}}=P_{\text {sat }}-\frac{\sigma}{r_{\max }}
\end{array}
$$

where $L_{\mathrm{g}}$ is the length of the grooves and $P_{\text {sat }}$ the saturation pressure. $r_{\max }$ is the maximum radius of curvature at the condenser. We showed in [18] that the maximum radius of curvature depends on the filling ratio, of the vapour space thickness and of the heat flux transferred by the FPHP. In this study, $r_{\max }$ is considered as an unknown that has to be determined from experimental data.

The rate of phase change introduced in equations (1) and (2) is calculated by a thermal model. A part of the heat is transferred by heat conduction in the wall and not by phase change at the liquid-vapour interface, due to heat diffusion in the FPHP wall.

\subsection{Thermal modelling}

The thermal model is a simplified version of the thermal model presented by Lefèvre et al. [13]. It takes into account longitudinal conduction in the wall, transversal conduction in the copper and the fluid, and phase change at the liquid-vapour interface. The FPHP is divided into several control volumes $i$ for which the energy balance equation is written (figure 2). The saturation temperature $T_{\text {sat }}$ is supposed to be constant and the wall temperature $T_{\mathrm{i}}$ is calculated for each control volume. A heat flux $q_{\mathrm{w}, \mathrm{i}}$ that depends on the location of the control volume in the FPHP, is imposed at the wall:

$$
q_{w, i}=\left\{\begin{array}{cl}
q_{\text {cond }}=-\frac{Q}{S_{\text {cond }}} & \text { at the condenser } \\
0 & \text { in the adiabatic zone } \\
q_{\text {evap }}=\frac{Q}{S_{\text {evap }}} & \text { at the evaporator }
\end{array}\right.
$$

where $Q$ is the total heat transferred by the system and $S_{\text {evap }}$ and $S_{\text {cond }}$ are the surface of the heat source and the heat sink respectively.

Two different thermal resistances are taken into account in the nodal model: a longitudinal thermal resistance $R_{\mathrm{th}, \text { long }}$ and a transversal thermal resistance $R_{\mathrm{th}, \mathrm{trans}, \mathrm{i}} \cdot R_{\mathrm{th}, \text { long }}$ is due to heat conduction in the wall of the FPHP along the $x$ direction. $R_{\mathrm{th}, \text { long }}$ is constant all along the system. $R_{\mathrm{th}, \mathrm{trans,i}}$ represents the thermal resistance due to transversal heat conduction in the wall and in the liquid and heat transfer by phase change at the liquid-vapour interface. As the liquid thickness and the liquid velocity are 
small, liquid convection is neglected in the grooves and heat transfer in the liquid is modelled by $2 \mathrm{D}$ heat conduction. In [13], a predictive model based on the liquid-vapour interface shape (i.e. the meniscus curvature radius calculated in the hydrodynamic model) was developed for both the condenser and the evaporator. In the evaporator, the top of the fin being dry, it was supposed to be adiabatic. In the condenser, a hydrodynamic model was developed to calculate the shape of the liquid, condensed on the fins. At the liquid-vapour interface, a heat transfer coefficient calculated from the gas kinetic theory [20] was introduced to take into account the high heat fluxes at the junction of the meniscus with the wall:

$$
h_{\text {evap } / \mathrm{cond}}=\frac{2 a}{2-a} \frac{\rho_{v} h_{l v}^{2}}{T_{\text {sat }}} \frac{1}{\sqrt{2 \pi \frac{\bar{R}}{\bar{M}} T_{\text {sat }}}}\left(1-\frac{P_{\text {sat }}}{2 \rho_{v} h_{l v}}\right)
$$

where $\bar{R}$ is the ideal gas constant, $\bar{M}$ the molar mass of the fluid and $a$ the accommodation coefficient.

As it was mentioned in [13], the evaporator and the condenser models are highly dependent on the accommodation coefficient, which is very difficult to determine experimentally. As a result this parameter is not referenced in the literature well. In [13], in similar experimental conditions, the variation of $\mathrm{R}_{\mathrm{th}, \mathrm{trans}, \mathrm{i}}$ was shown to be rather small in both the evaporator and the condenser. Therefore, in this study, $R_{\mathrm{th}, \text { trans,i }}$ parameters are supposed to be constant in the evaporator and the condenser : $R_{\mathrm{th}, \text { trans,evap }}$ and $R_{\mathrm{th}, \text { trans,cond }}$ are calculated by a parameter estimation method based on the temperature measurement data. Detailed investigations of evaporation and condensation processes specific to grooved flat heat pipes are presented in [23].

The energy balance in each control volume leads to a set of equations written as follows:

$$
\frac{T_{i-1}+T_{i+1}}{R_{\text {th }, \text { long }}}-T_{i}\left(\frac{2}{R_{\text {th }, \text { long }}}+\frac{1}{R_{\text {th }, \text { trans }, i}}\right)+\frac{T_{\text {sat }}}{R_{t h, t r a n s, i}}+q_{w, i}=0
$$

As the FPHP is divided in $n$ nodes, we obtain a set of $n$ equations with $n+4$ unknowns: $\mathrm{n}$ temperatures at each node, the saturation temperature, the longitudinal thermal resistance and the transversal thermal resistances at the condenser and at the evaporator.

A parameter estimation method (least square minimisation) was developed for the determination of the longitudinal thermal resistance: temperature measurements, obtained when the FPHP is empty for different heat transfer rates, are compared with the thermal model, while $R_{\mathrm{th}, \mathrm{trans}, \mathrm{i}}$ is set to infinity. A similar parameter estimation method was developed for the determination of the saturation temperature and the transversal thermal resistances at the condenser and at the evaporator: temperature measurements, obtained when the FPHP is filled for different heat transfer rates, are compared with the thermal model. 
The thermal model is used to calculate the heat flux by evaporation or condensation, which is a parameter of the hydrodynamic model:

$$
q_{i}=\frac{T_{i}-T_{\text {sat }}}{R_{\text {th,trans }, i}}
$$

\section{An experimental bench for the measurement of the capillary pressure and the temperature}

\subsection{Experimental set-up}

The flat heat pipe under investigation is shown in figure 3. It was already described in previous articles $[17,18]$ to which the reader should refer for further details. Its capillary structure, of dimensions $70 \times 90 \mathrm{~mm}^{2}$, is made of 88 longitudinal micro-grooves, machined in a copper plate. The grooves are connected at the condenser. Each groove has a rectangular cross-section of height and width equals to $400 \mu \mathrm{m}$. The distance between two grooves is equal to $400 \mu \mathrm{m}$. The wall thickness under the grooves is equal to $3 \mathrm{~mm}$. The FPHP is hermetically sealed on its upper face with a transparent plate, which allows the observation of the liquid/vapour menisci in the grooves. The vapour space thickness can vary by changing the thickness of a nitrile ring. The heat source is a heated copper block of dimensions $70 \times 20 \mathrm{~mm}^{2}$ supplied by a $0-220 \mathrm{~V}$ AC power supply. The heat sink is a water heat exchanger of dimensions $30 \times 70 \mathrm{~mm}^{2}$. The water flow rate is constant and the inlet temperature is controlled by means of a thermostatic bath in order to have a constant working temperature when the heat input increases. The heat source and the heat sink are separated by an adiabatic area of length equal to $40 \mathrm{~mm}$.

Two series of nine calibrated thermistors (uncertainty lower than $0.2 \mathrm{~K}$ ) are located symmetrically along the FPHP wall. The working temperature of the FPHP is the saturation temperature $T_{\text {sat }}$, which is determined as the mean temperature of the six thermistors located in the middle of the adiabatic zone.

A confocal microscope is used to measure the meniscus curvature radius in the grooves, the FPHP being in horizontal orientation. The confocal microscope is a STIL Micromesure 2 system. The optical sensor has a nominal measuring range of $350 \mu \mathrm{m}$. More details about the use of confocal microscopy for the study of FPHP are available in $[4,18,19]$.

The profile of the liquid-vapour interface is measured every $5 \mathrm{~mm}$ along several grooves (between 10 and 14 grooves, depending on the experiment) located at the centre of the FPHP. Meniscus curvature radii are calculated from the experimental data by a non linear least square method [24]. This method allows the calculation of the uncertainty for the estimated curvature radii from the 
uncertainty for the profile measurement. This uncertainty is small compared to the physical dispersion observed between the grooves in working conditions. Therefore, the measurements obtained on several grooves are averaged to increase the accuracy of the method as it is presented in section 3.3.

It has to be noticed that nucleate boiling occurs for rather small heat transfer rates [17], which prevents the interface visualization for high heat transfer rates. Nevertheless, boiling does not constitute an operating limit as it is shown in section 4.

\subsection{Validation of the experimental method in non working conditions}

In order to validate the experimental method, measurements were realised in non working conditions, the FPHP being tilted. Figure 4 and 5 present the evolution of the meniscus curvature radii and the resulting capillary pressures $\left(P_{\text {cap }}=\sigma r\right)$ respectively. The FPHP is filled with n-pentane, and two inclination angles are tested $\left(\theta=2.5^{\circ}\right.$ and $\left.\theta=5^{\circ}\right)$. The uncertainty for the slope angle is equal to $0.1^{\circ}$. In these conditions, the capillary pressure gradient is theoretically equal to the hydrostatic pressure in the liquid:

$$
\frac{d P_{c a p}}{d x}=\rho_{l} g \sin \theta
$$

with $\theta$ the angle between the FPHP and the horizontal. Solid lines present the theoretical evolution of the capillary pressure. A good agreement is observed between experimental and theoretical results. It validates our experimental method.

\subsection{Experimental results in working conditions}

The FPHP was studied in a variety of experimental conditions and a significant experimental database was created (table 2). Experimental parameters are the vapour space thickness $h_{\mathrm{v}}$, the inclination angle $\theta$, the filling ratio $f_{\mathrm{r}}$ and the heat transferred by the system $Q$. N-pentane at a saturation temperature equal to $40^{\circ} \mathrm{C}$ is used as working fluid. In [4], the FPHP was tested with methanol. In the present study, n-pentane was chosen because it is chemically compatible with the transparent plate, which is made of polycarbonate. In [4], it was in borosilicate but thermal mechanical constraints were too high in the present experiments to use borosilicate. Some of the results presented in this section were already presented and detailed in previous articles [18,25]. New results, obtained in tilted positions are presented too.

Figure 6 presents an example of curvature radius measurements for $Q=88 \mathrm{~W}$ and $f_{r}=13 \%$ in several grooves. Figure 7 is the capillary pressure deduced from the meniscus measurements. To facilitate and reduce the presentation of the hydrodynamic measurements, the experimental results obtained by confocal microscopy are presented in the form of the mean capillary pressure gradient 
$\Delta \mathrm{Pcap} / \Delta \mathrm{x}$ in the adiabatic zone $(40 \mathrm{~mm}<\mathrm{x}<80 \mathrm{~mm})$. In this zone, the slope can be considered constant because the mass flow rate and the liquid cross section are almost constant. $\Delta \mathrm{Pcap} / \Delta \mathrm{x}$ (i.e. the slope of the straight lines in figure 7) is calculated for each groove as well as the associated uncertainty. Figure 6 shows that an important physical dispersion exists between the grooves. It has to be noted that this example is one of the most dispersed experiment. This dispersion is randomly distributed and can thus not be explained by non uniform distribution in the heat flux. Among possible explanations, one might suggest that a non homogeneous distribution of the roughness of the copper could modify the apparent contact angle of the liquid on the wall and thus the average value of the meniscus curvature radius in the groove.

Nevertheless, the slopes of the calculated capillary pressure are not as much scattered and thus the uncertainty for the capillary pressure gradient (obtained by a weighted average of each groove with $95 \%$ confidence level) is rather small compared to that for the meniscus curvature radii at a given location in the FPHP. Finally, the method was validated in non working conditions and even though the measurements are scattered, a good agreement is found between the experimental results and the

model. In table 2, the uncertainty for the capillary pressure gradient is given for each experiment.

The temperature measurements are presented in the form of a thermal resistance $r_{\mathrm{th}}$, which is obtained by calculating the ratio of the maximum temperature difference in the FPHP wall and the heat transfer rate transferred by the system.

This database can be enlarged with the results presented by Rullière et al. in [4]. The results were obtained with a copper FPHP of larger dimensions (90 $\mathrm{mm}$ x $230 \mathrm{~mm}$ ), designed for the cooling of a PEMFC fuel cell. The capillary structure is made of 109 longitudinal grooves of $400 \mu \mathrm{m}$ depth and $400 \mu \mathrm{m}$ width. Experimental results were obtained with methanol as working fluid at a saturation temperature of $70^{\circ} \mathrm{C}$.

Table 3 presents the experimental results obtained with this FPHP for $Q$ equal to $85.5 \mathrm{~W}, 119.7$ $\mathrm{W}$ and $153.9 \mathrm{~W}$. The mean capillary pressure is calculated on a length equal to $80 \mathrm{~mm}$ around the adiabatic area, where the capillary pressure slope can be considered constant.

The thermal resistance is much lower for the FPHP tested by Rullière et al. [4], which is due to the size of the system and of the evaporating and condensing area. In the present FPHP, as the length is rather small, a non negligible part of the heat (about $50 \%$ ) is transferred by heat conduction through the wall whereas it was lower than $5 \%$ with the FPHP tested by Rullière et al. [4].

\section{Comparison of the models with the experimental database}

In this section, the experimental data base is compared with the models previously described. The thermal model is solved using the temperature measurements obtained when the FPHP is filled and 
empty. The temperature field in the wall and the evaporation and condensation heat fluxes are calculated. Mass flow rates of evaporation or condensation are introduced in the hydrodynamic model to calculate the liquid and vapour pressures and velocities, as well as the meniscus curvature radius along the grooves.

\subsection{Experimental validation of the hydrodynamic model}

Figure 8 represents the mean capillary pressure gradients. Experimental results are plotted versus numerical results obtained in similar conditions. The estimated uncertainties (confidence level of $95 \%$ ) are given in each experimental condition except for Rullière's results. In all the cases, a good agreement is observed between experimental and numerical results. The numerical model tends to slightly overestimate the capillary pressure gradient in the system. The mean relative error of the model is about $15 \%$.

\subsection{Discussions on the interfacial shear stress}

Interfacial shear stress is often considered has being an important issue in modelling FPHP $[14,26,27]$. In the present hydrodynamic model, interfacial shear-stress was calculated considering the liquid as motionless for the vapour. We have tested two other assumptions, i.e. fixed interface and free interface, to check the sensitivity on the model (Figure 9). The assumption of a fixed liquid-vapour interface leads to an important overestimation of the capillary pressure gradient in the system. The results obtained with no shear stress (free interface) are close to those obtained with the assumption of a static liquid compared to the vapour. This result shows that in all these experimental conditions, modelling the interfacial shear stress is not a real issue even for a rather small vapour space thickness of $1 \mathrm{~mm}$.

It has to be noted that the experimental conditions are close to those encountered in electronic cooling [1]. A thinner vapour space would lead to a higher vapour velocity and thus to the increase of the interfacial shear stress. Therefore, it could be interesting to perform a similar analysis with smaller vapour spaces. Nevertheless, we have shown in a previous paper [18] that reducing the vapour space thickness has a huge influence on the maximum capillary pressure that a grooved capillary structure can sustain. When the vapour space is small, a meniscus appears between the two plates of the FPHP. The curvature radius of this meniscus decreases with the decrease of the vapour space thickness. As a result, the liquid pressure at the condenser is small compared to that with a high vapour space and thus the capillary pressure gradient between the condenser and the evaporator is reduced. For example, in the present experimental conditions, a vapour space of $1 \mathrm{~mm}$ leads to $35 \%$ decrease of the maximum capillary pressure with respect to an infinite vapour space thickness. Therefore, vapour space thicknesses above $1 \mathrm{~mm}$ would not allow understanding the influence of the interfacial shear stress. 


\subsection{Effect of nucleate boiling on the capillary limit}

We showed in previous papers [17,19], that nucleate boiling occurs in the evaporator of FPHPs for rather small heat fluxes. In the present experiment, nucleate boiling was observed for heat fluxes higher than $3 \mathrm{~W} / \mathrm{cm}^{2}$ and until $18 \mathrm{~W} / \mathrm{cm}^{2}$. We pointed out in [17] that this phenomenon does not prevent an efficient operation of the system and conversely increases its thermal performance. However, the experimental results were not sufficient to conclude if the dryout observed at high heat fluxes was due to nucleate boiling or to the capillary limit. In the present work, new measurements were made to cope with this question.

The goal of these experiments is to reach the dryout of the evaporator for different experimental conditions (i.e. with and without nucleate boiling) and to compare the maximum heat fluxes in the different conditions. For that purpose, the FPHP is tilted in unfavourable position, i.e. the evaporator being above the condenser. As a result, dryout is observed for smaller heat fluxes than in horizontal orientation, because of the gravity force. There is a maximum angle for which volume forces are equal to the maximum capillary pressure that the FPHP can sustain. For that maximum angle, the FPHP is unable to transfer any heat without the occurrence of dryout at the evaporator. For decreasing dryout angles, the maximum heat flux that the FPHP can sustain increases. Thus, for high dryout angles, nucleate boiling is not encountered and it appears and grows in intensity for decreasing dryout angles.

Figure 10 shows the evolution of the dryout angle versus the dryout heat flux. The FPHP $\left(h_{\mathrm{v}}=2\right.$ $\mathrm{mm})$ is filled with methanol $\left(f_{\mathrm{r}}=10 \%\right)$ at a saturation temperature of $60{ }^{\circ} \mathrm{C}$. The maximum dryout angle is equal to $8^{\circ}$. Nucleate boiling occurs for heat fluxes higher than $3 \mathrm{~W} / \mathrm{cm}^{2}$. The thermal resistance of the FPHP, $r_{\text {th }}$ calculated for a heat flux slightly lower than the dryout heat flux is shown in figure 11. The thermal resistance decreases when the heat flux increases: this is due to the effect of nucleate boiling that improves heat transfer inside the FPHP.

The capillary limit $\Delta P_{\text {cap }}$ (i.e. the maximum capillary pressure gradient that the capillary pressure can sustain) is equal to the sum of pressure drops in liquid flow $\Delta P_{1}$ and vapour flow $\Delta P_{\mathrm{v}}$ and the hydrostatic pressure $\Delta P_{\mathrm{g}}$.

$$
\Delta P_{c a p}=\Delta P_{g}+\Delta P_{v}+\Delta P_{l}
$$

When nucleate boiling occurs, confocal microscopy can not be used to measure the capillary pressure gradient. Therefore, in this section, pressure drops in the liquid and the vapour are calculated by means of the hydrodynamic model in the same conditions as the experiment. 
Figure 12 represents $\Delta P_{\text {cap }}$ and $\Delta P_{\mathrm{g}}$ versus the dryout heat flux. Uncertainties are calculated by taking into account the numerical model uncertainty (about 15\%, cf. section 3.1) and the uncertainty on the tilt angle $\left(0.1^{\circ}\right)$. The uncertainty increases when the heat flux increases because the part of the capillary pressure due to liquid and vapour pressure drops increases. Horizontal lines are theoretical capillary pressures. They are calculated considering a meniscus curvature radius equal to half the width of a groove at the evaporator and a curvature radius at the condenser $r_{0}$ either equal to an infinite value or to $2 \mathrm{~mm}$. This last assumption was discussed in [18] for a FPHP in horizontal location.

This result shows that the capillary pressure variation is almost constant, whatever the inclination of the FPHP. Thus, the dryout of the evaporator is due to the capillary limit and not to a limit induced by the occurrence of nucleate boiling in the grooves. It has to be noted that the capillary pressure for the highest heat flux $\left(18 \mathrm{~W} / \mathrm{cm}^{2}\right)$ is slightly lower than for the other heat fluxes. Nevertheless, as the FPHP is in horizontal orientation, the vapour space thickness limits the maximum meniscus curvature at the condenser, and thus the maximum capillary pressure that the grooves can sustain, as it was shown in [18]. This result is important because it questions a fundamental feature of the heat pipe literature for grooved capillary structure. It has to be noticed that this result was obtained in a $1 \mathrm{~g}$ gravity field. Would these observations be similar in space, a condition for which the vapour would not escape from the grooves easily? Furthermore, it would be interesting to perform such an experiment with other capillary structures like sintered powder wicks or meshes.

\section{Conclusion}

An experimental database, obtained with a grooved FPHP made of copper and filled with npentane is presented. Temperature and meniscus curvature radius measurements were made for different experimental parameters (vapour space thickness, filing ratio, heat transfer rate, slope of the FPHP). This database was completed with results of Rullière et al. [4] for a FPHP made of copper and filled with methanol. Experimental results are compared to results of coupled thermal and hydrodynamic models. A good agreement is found between the hydrodynamic model and the experimental results. The effect of the interfacial thermal resistance is found to be negligible in all the experimental conditions. An experiment conducted in non horizontal location shows that the presence of nucleate boiling in the system does not constitute a limit as it is generally stated in the literature. In all the cases, dryout of the evaporator is due to the capillary limit.

The present thermal model is not a predictive model, based on the shape of the liquid-vapour interface as in [13]. Nevertheless, such an approach is highly influenced by the accommodation coefficient, which is poorly studied in the literature. This a key point for future works on FPHP because thermal models are highly functions of this parameter. Furthermore, nucleate boiling has to be introduced in FPHP thermal models. 


\section{Acknowledgments}

This work was supported by the GIP-ANR in the frame of the non-thematic project “INTENSIFILM” No ANR-06-BLAN-0119-03.

\section{References}

[1] M. Lallemand, F. Lefèvre, Micro/mini heat pipes for the cooling of electronic devices, 13th International Heat Pipe Conference, Shanghaï, China, 2004, pp. 12-22.

[2] J. P. Longtin, B. Badran, F. M. Gerner, A one-dimensional model of a micro heat pipe during steady-state operation. J. Heat Transfer, 116 (1994) 709-715

[3] S. Launay, V. Sartre, M. B. H. Mantelli, K. V. de Paiva, M. Lallemand, Investigation of a wire plate micro heat pipe array. Int. J. Therm. Sci., 43 (2003) 499-507

[4] R. Rullière, F. Lefèvre, M. Lallemand, Prediction of the maximum heat transfer capability of two-phase heat spreaders - Experimental validation. Int. J. Heat Mass Transfer, 50 (2007) 12551262

[5] M. Ivanova, A. Lai, C. Gillot, N. Sillon, C. Shaeffer, F. Lefèvre, M. Lallemand, E. Fournier, Design, fabrication and test of silicon heat pipes with radial microcapillary grooves. ITHERM'2006, San Diego, 30 mai - 2 Juin (2006)

[6] F. Lefèvre, R. Revellin, M. Lallemand, Theorical analysis of two-phase heat spreaders with different cross-section micro grooves. 7th IHPS, Jeju, Korea, (2003) 97-102

[7] B. Suman, P. Kumar, An analytical model for fluid flow and heat transfer in a micro-heat pipe of polygonal shape. Int. J. Heat Mass Transfer, 48 (2005) 4498-4509

[8] B. Suman, S. De, S. DasGupta, Transient modeling of micro-grooved heat pipe. Int. J. Heat Mass Transfer, 48 (2005) 1633-1646

[9] Y. X. Wang, G. P. Peterson, Analysis of wire-bonded micro heat pipe arrays. J. Thermophysics Heat Transfer, 16, 3 (2002) 346-355

[10] F. Lefèvre, M. Lallemand, Coupled thermal and hydrodynamic models of flat micro heat pipes for the cooling of multiple electronic components. Int. J. Heat Mass Transfer, 49 (2006) $1375-1383$

[11] N. Zhu, K. Vafai, Vapor and liquid flow in a asymmetrical flat plate heat pipe : a three dimensional analytical and numerical investigation. Int. J. Heat Mass Transfer, 41, 1 (1998) 159174

[12] B. Xiao, A. Faghri, A three-dimensional thermal-fluid analysis of flat heat pipes, Int. J. Heat Mass Transfer 51 (2008) 3113-3126

[13] F. Lefèvre, R. Rullière, G. Pandraud, M. Lallemand, Prediction of the temperature field in flat plate heat pipes with micro-grooves - Experimental validation. Int. J. Heat Mass Transfer, 51 (2008) 4083-4094

[14] D. Khrustalev, A. Faghri, Coupled liquid and vapor flow in miniature passages with micro grooves, J. Heat Transfer 121 (1999) 729-733.

[15] S.J. Kim, J.K. Seo, K.H. Do, Analytical and experimental investigation on the operational characteristics and the thermal optimization of a miniature heat pipe with a grooved wick structure, Int. J. Heat Mass Transfer 46 (2003) 2051-2063.

[16] R. Hopkins, A. Faghri, D. Khrustalev, Flat miniature heat pipes with micro capillary grooves, J. Heat Transfer, 121 (1999) 102-109 
[17] S. Lips, F. Lefevre, J. Bonjour, Nucleate boiling in a flat grooved heat pipe. Int. J. Therm. Sci., 48 (2009) 1272-1278

[18] S. Lips, F. Lefèvre, J. Bonjour, Combined effects of the filling ratio and the vapour space thickness on the performance of a flat plate heat pipe. Int. J. Heat Mass Transfer, 53 (2010) 11131121

[19] F. Lefevre, R. Rullière, S. Lips, J. Bonjour, Confocal microscopy applied to capillary film measurements in a radial flat plate heat pipe made of silicon. J. Heat Transfer, accepted $16^{\text {th }}$ of july 2009

[20] V. P. Carrey, Liquid-vapour phase change phenomena : an introduction to the thermophysics of vaporization and condensation process in heat transfer equipment. Bristol, by Taylor \& Francis, (1992) 645 p.

[21] J. G. Collier, J. R. Thome, Convective boiling and condensation. Oxford University Press, Oxford, UK, 3rd edition (1996) $591 \mathrm{p}$.

[22] A. Faghri, Heat pipe science and technology, Taylor and Francis, London, 1995

[23] F. Lefèvre, S. Lips, J. Bonjour, Investigation of evaporation and condensation processes specific to grooved flat heat pipes, Frontiers in Heat Pipes (FHP), 1 (2010) 023001.

[24] J. V. Beck, K.J. Arnold, Parameter estimation in engineering and science. Wiley and sons, New York (1977)

[25] S. Lips, F. Lefèvre, J. Bonjour, Thermal and hydrodynamic study of a flat plate heat pipe, Experimental Heat Transfer, Fluid Mechanics and Thermodynamics ExHFT-7, Krakow, Poland, 28 juin - 03 juillet (2009)

[26] G. E. Schneider, R. Devos, Non-dimensional analysis for the heat transport capability of

axially grooved heat pipes including liquid/vapor interaction. American Institute of Aeronautics and Astronautics, 18th Aerospace Sciences Meeting, No. 80-0214 (1980)

[27] R. Jacolot, C. Romestant, V. Ayel, Y. Bertin, J. Hugon, Visualisation et analyse expérimentale du comportement du fluide dans un caloduc rainuré. Congrès Français de Thermique SFT, Iles des ambiez, 29 mai - 1 Juin (2007) 143-148 


\section{List of figure captions}

Figure 1: Volume control

Figure 2: Thermal nodal network

Figure 3: Schematic of the FPHP

Figure 4: Meniscus curvature radii measured for two tilt angles

Figure 5: Comparison between hydrostatic and capillary pressures

Figure 6: Dispersion of the measured meniscus curvature radii $\left(Q=88 \mathrm{w} ; f_{\mathrm{r}}=13 \%\right)$

Figure 7: Dispersion of the capillary pressures $\left(Q=88 \mathrm{~W} ; f_{\mathrm{r}}=13 \%\right)$

Figure 8: Comparison between experimental and numerical hydrodynamic results

Figure 9: Comparison between experimental and numerical hydrodynamic results with different assumptions about the interfacial shear stress

Figure 10: Tilted angle at the dry-out heat flux

Figure 11: $\mathbf{R}_{\text {th }}$ at the dry-out heat flux

Figure 12: Capillary and hydrostatic pressures versus the dry-out heat flux

List of table captions

Table 1 : Terms taken into account in the momentum equation by different authors

Table 2 : Experimental database with the present FPHP

Table 3 : Experimental results of Rullière et al. (calculated from [4]) 


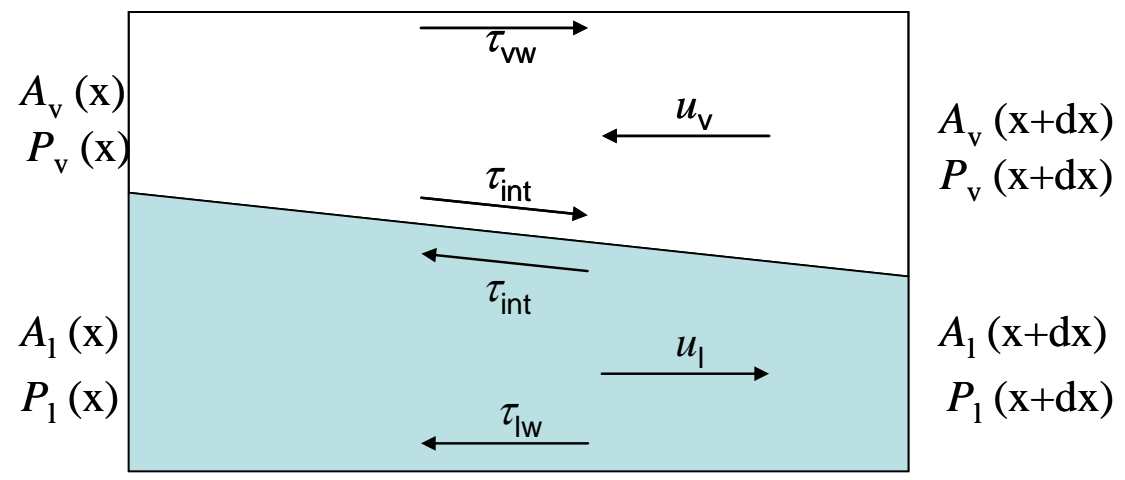

Figure 1: Volume control 


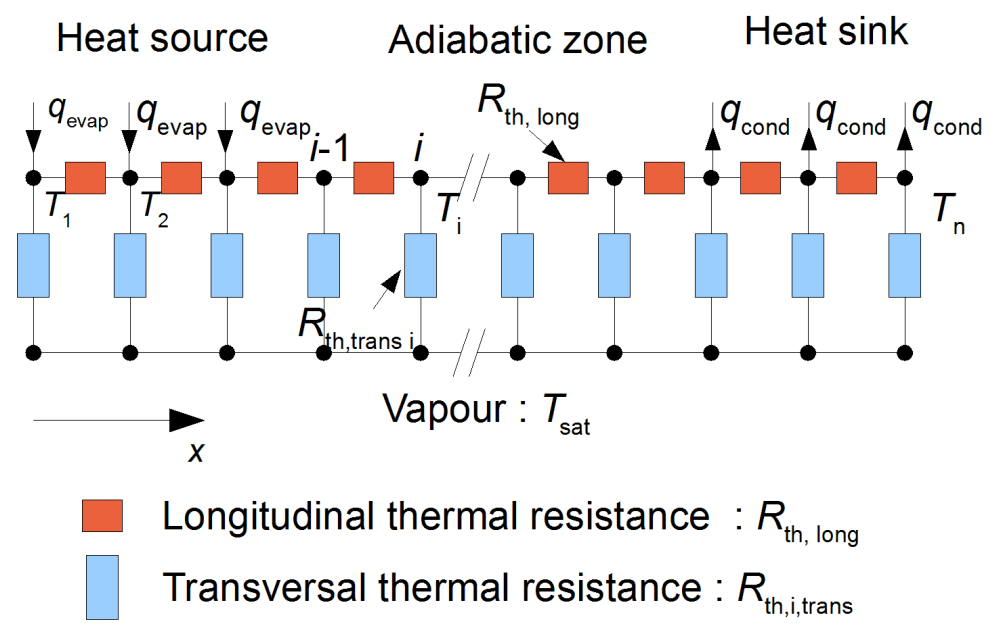

Figure 2: Thermal nodal network 


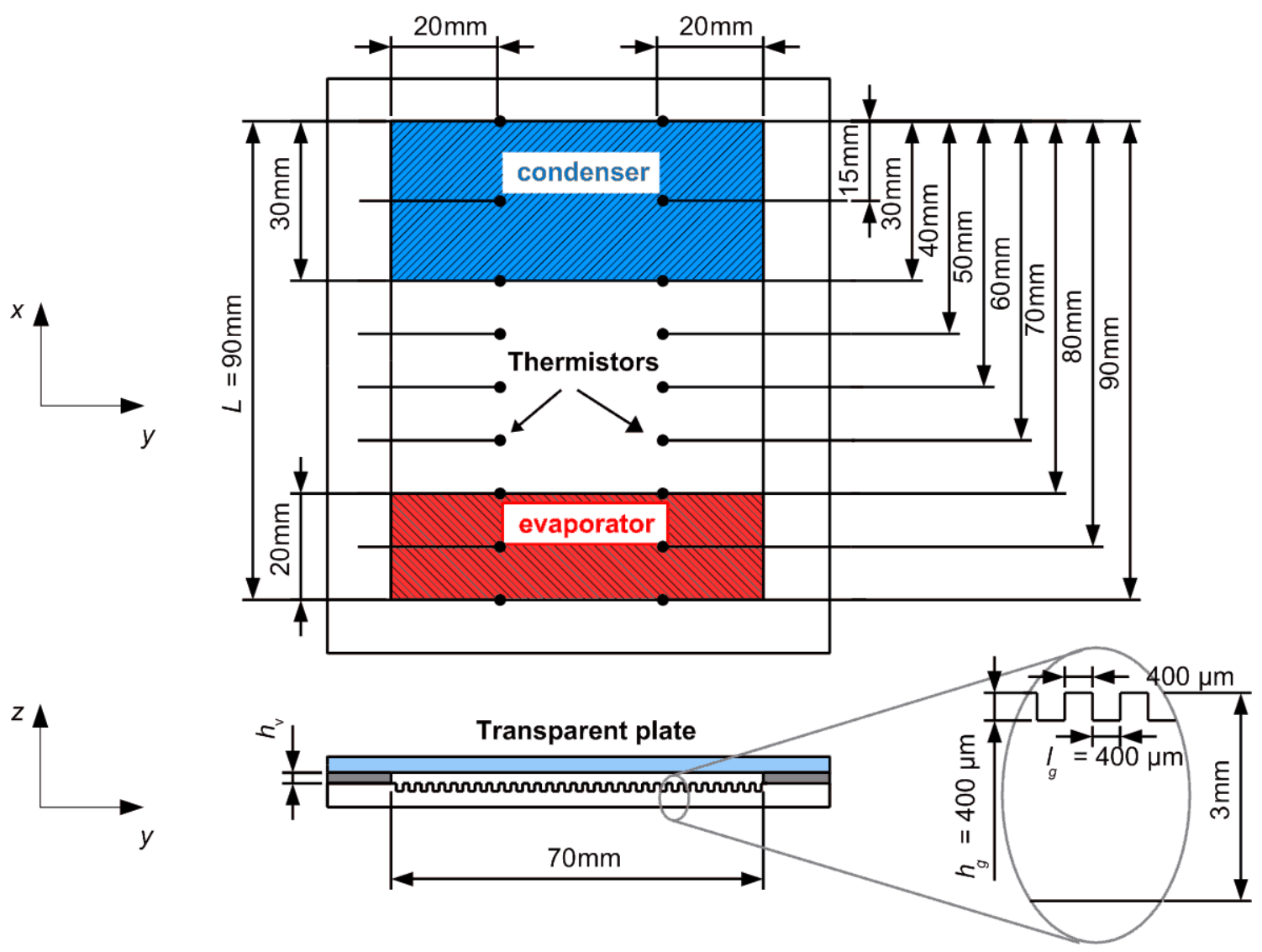

Figure 3: Schematic of the FPHP 


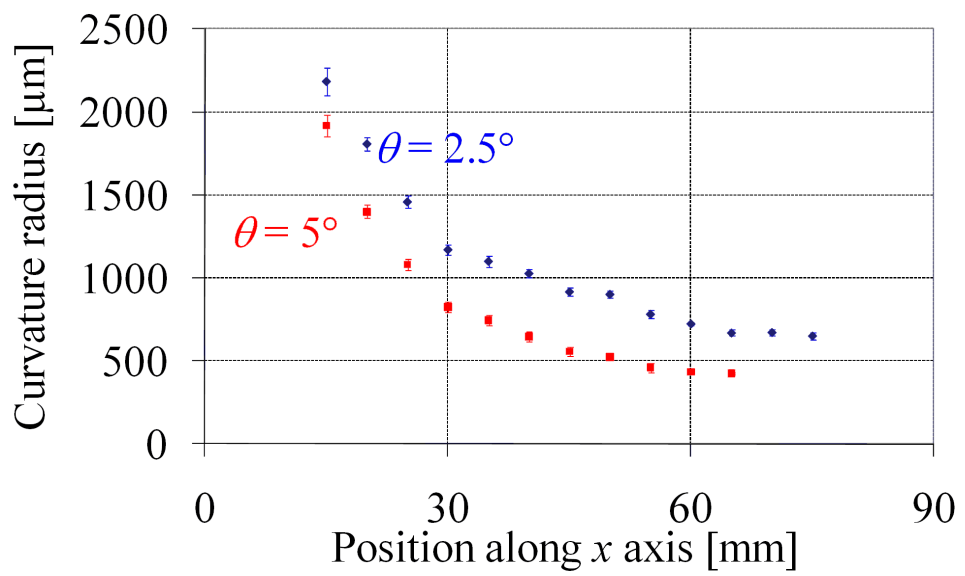

Figure 4: Meniscus curvature radii measured for two tilt angles 


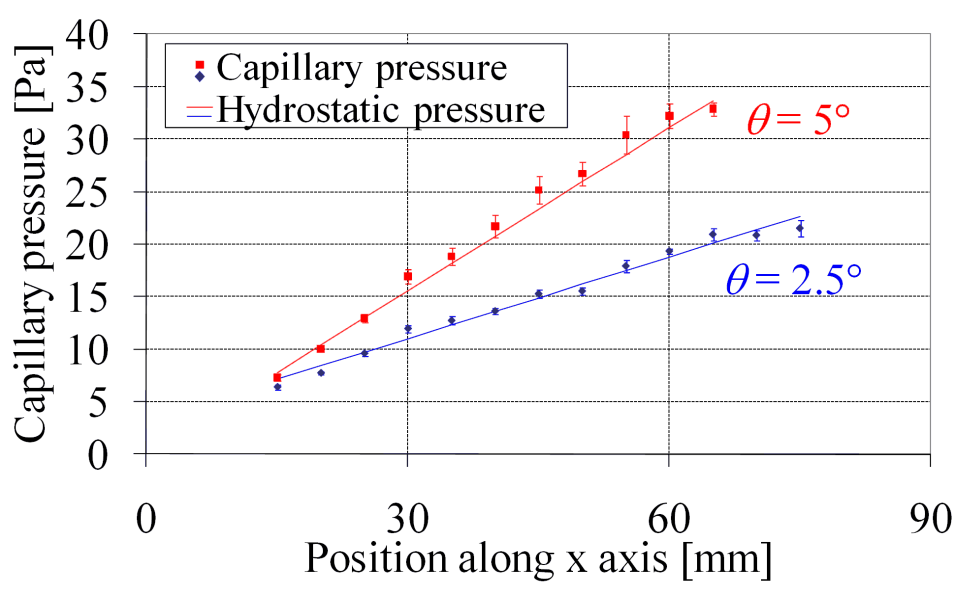

Figure 5: Comparison between hydrostatic and capillary pressures 


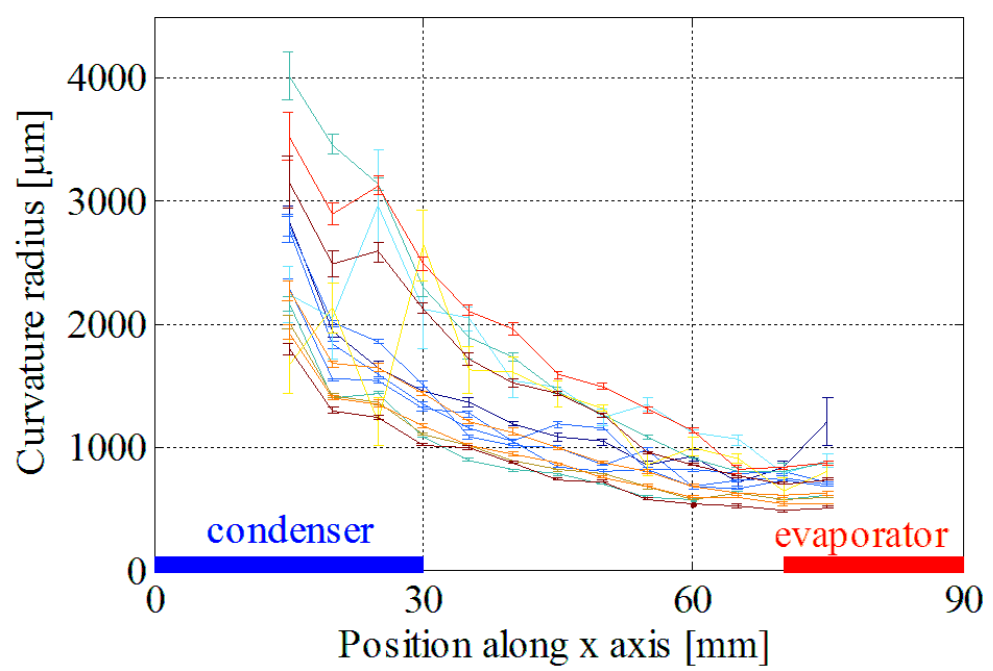

Figure 6: Dispersion of the measured meniscus curvature radii $\left(Q=88 \mathrm{~W} ; f_{\mathrm{r}}=13 \%\right)$ 


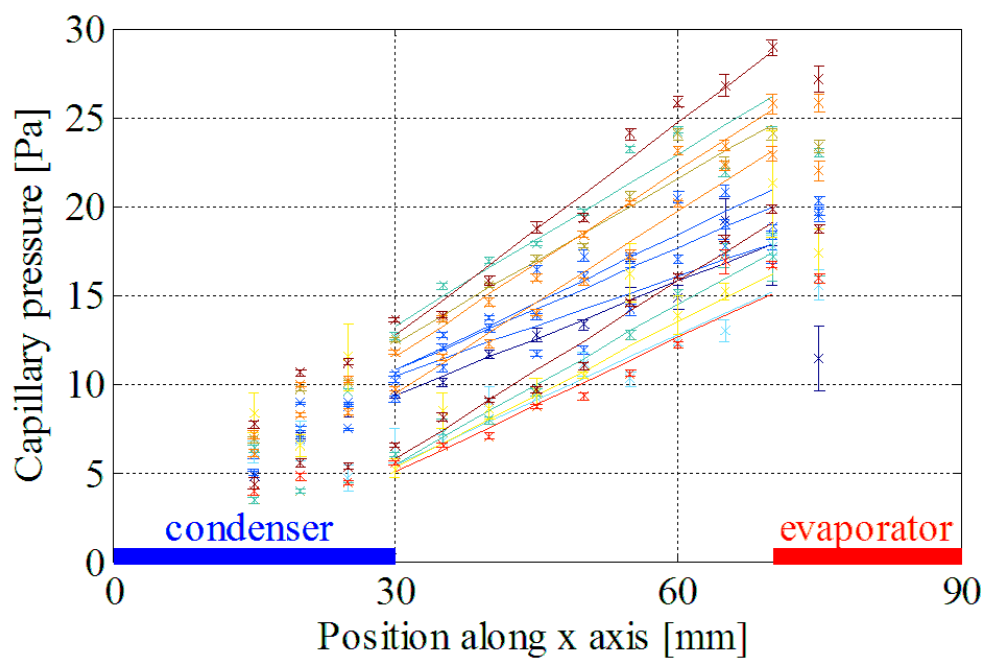

Figure 7: Dispersion of the capillary pressures $\left(Q=88 \mathrm{~W} ; f_{\mathrm{r}}=13 \%\right)$ 


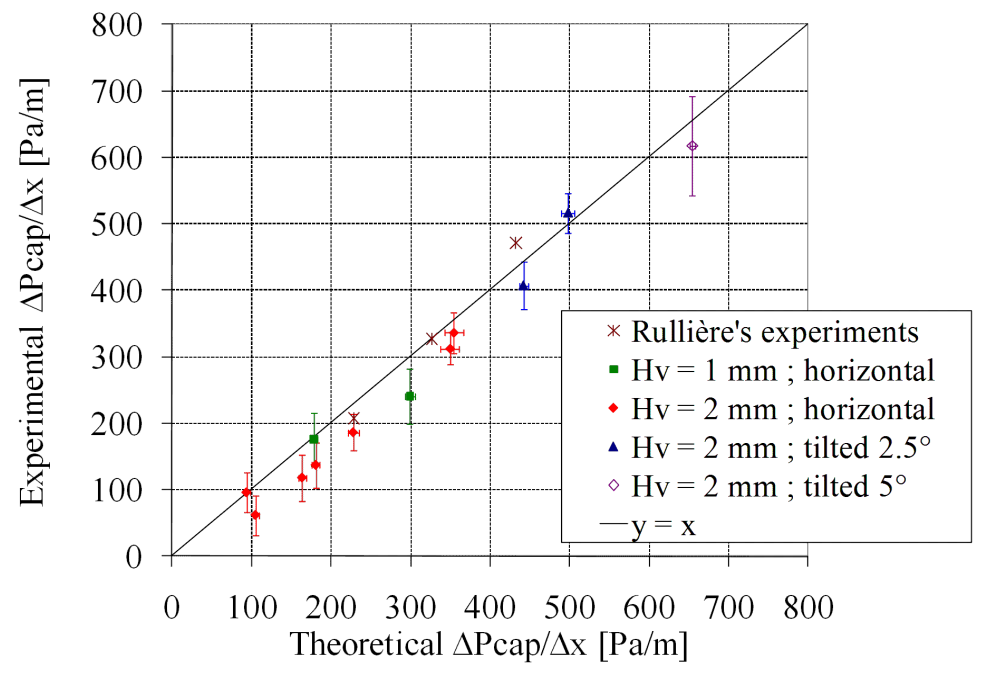

Figure 8: Comparison between experimental and numerical hydrodynamic results 


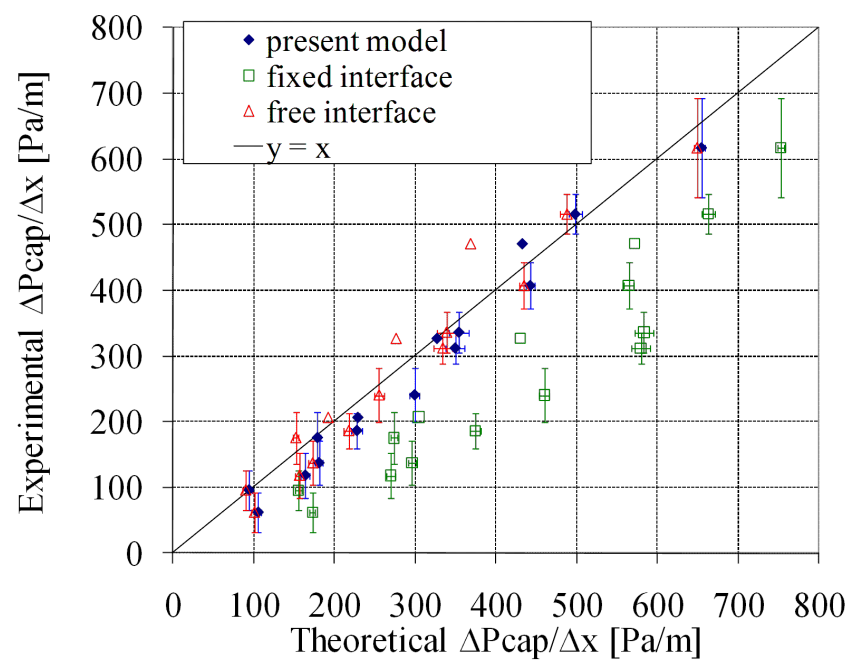

Figure 9: Comparison between experimental and numerical hydrodynamic results with different assumptions about the interfacial shear stress 


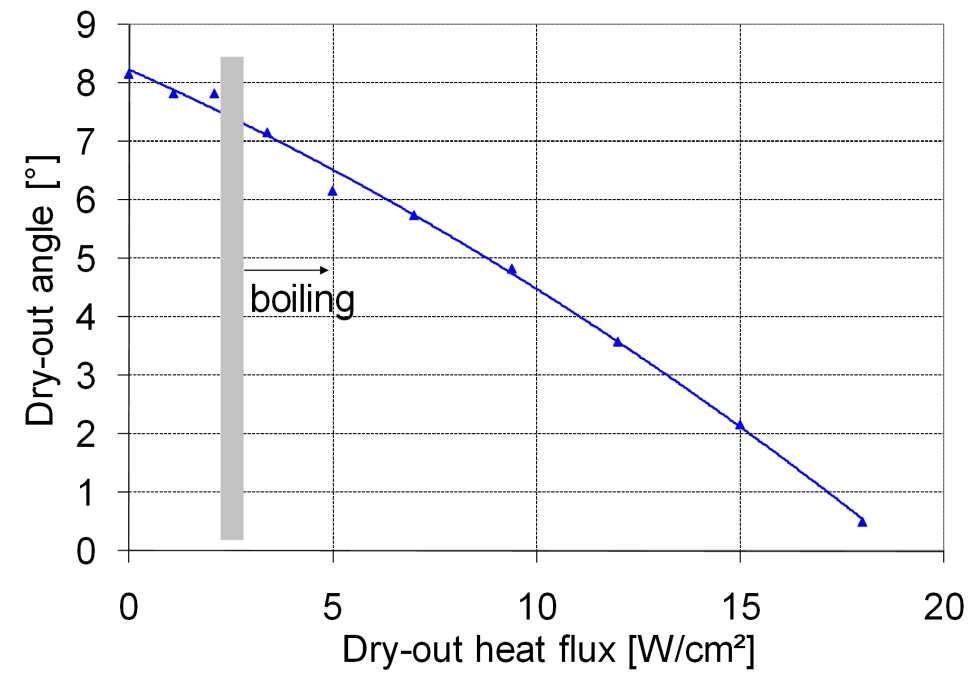

Figure 10: Tilted angle at the dry-out heat flux 


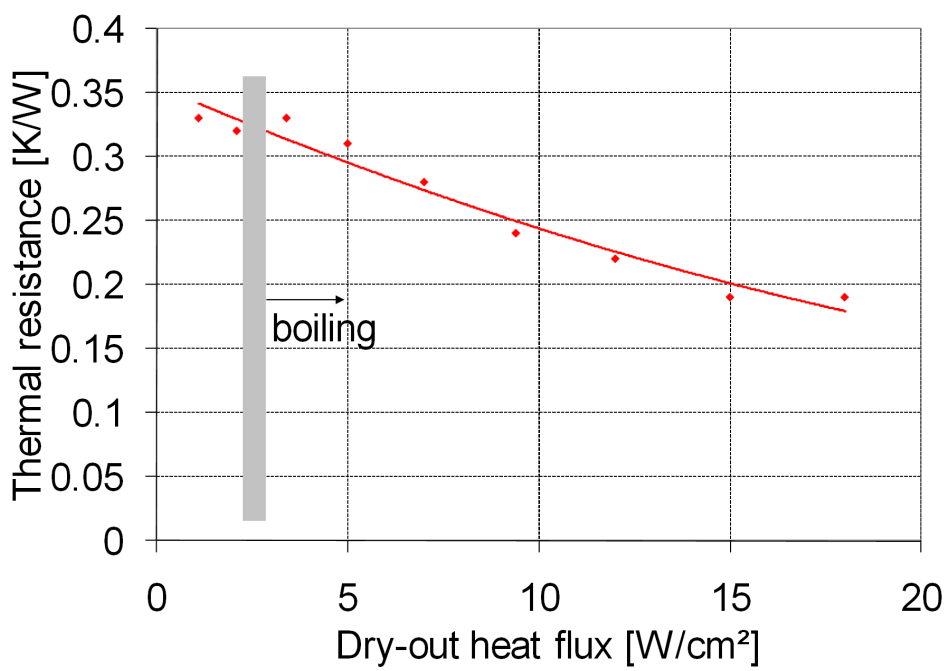

Figure 11: $\mathrm{R}_{\mathrm{th}}$ at the dry-out heat flux 


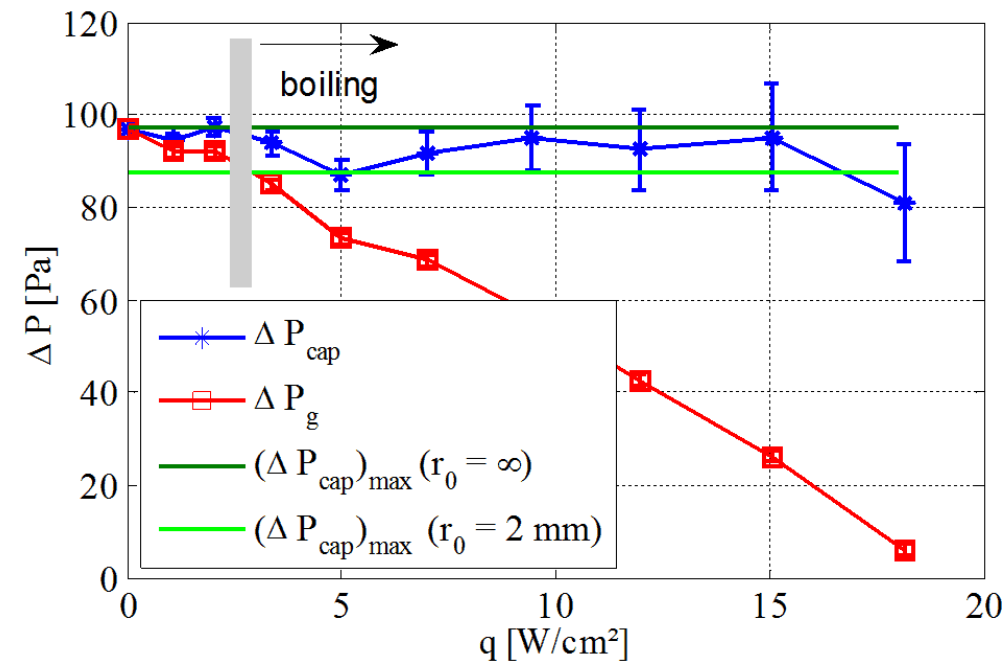

Figure 12: Capillary and hydrostatic pressures versus the dry-out heat flux 
Table 1 : Terms taken into account in the momentum equation by different authors

\begin{tabular}{|c|c|c|c|c|c|c|c|c|c|}
\cline { 2 - 10 } Author & $(\mathbf{1})$ & $\mathbf{( 2 )}$ & $\mathbf{( 3 )}$ & $\mathbf{( 4 )}$ & $\mathbf{( 5 )}$ & $\mathbf{( 6 )}$ & $\mathbf{( 7 )}$ & $\mathbf{( 8 )}$ & $\mathbf{( 9 )}$ \\
\hline Longtin et al. [2] & $\mathrm{X}$ & $\mathrm{X}$ & $\mathrm{no}$ & $\mathrm{no}$ & $\mathrm{no}$ & $\mathrm{X}$ & $\mathrm{X}$ & $\mathrm{X}$ & negl. \\
\hline Wang et Peterson [9] & $\mathrm{X}$ & $\mathrm{X}$ & no & no & no & $\mathrm{X}$ & $\mathrm{X}$ & $\mathrm{X}$ & no \\
\hline Launay et al.[3] & $\mathrm{X}$ & $\mathrm{X}$ & $\mathrm{no}$ & no & no & $\mathrm{X}$ & $\mathrm{X}$ & $\mathrm{X}$ & no \\
\hline Lefèvre et al. [6] & $\mathrm{X}$ & $\mathrm{X}$ & $\mathrm{X}$ & no & no & $\mathrm{X}$ & $\mathrm{X}$ & $\mathrm{X}$ & no \\
\hline Ivanova et al.[5] & $\mathrm{X}$ & $\mathrm{X}$ & $\mathrm{X}$ & no & no & no & $\mathrm{X}$ & $\mathrm{X}$ & no \\
\hline Suman et al. [7,8] & $\mathrm{no}$ & $\mathrm{X}$ & $\mathrm{no}$ & no & no & $\mathrm{X}$ & $\mathrm{X}$ & $\mathrm{no}$ & no \\
\hline Rullière et al. [4] & $\mathrm{X}$ & $\mathrm{X}$ & $\mathrm{X}$ & $\mathrm{X}$ & no & no & $\mathrm{X}$ & $\mathrm{X}$ & no \\
\hline Collier [21]* & $\mathrm{X}$ & $\mathrm{X}$ & $\mathrm{no}$ & no & no & $\mathrm{X}$ & $\mathrm{X}$ & $\mathrm{X}$ & $\mathrm{X}$ \\
\hline Carey [20]* & $\mathrm{X}$ & $\mathrm{X}$ & $\mathrm{X}$ & no & no & $\mathrm{X}$ & $\mathrm{X}$ & $\mathrm{X}$ & $\mathrm{X}$ \\
\hline
\end{tabular}

* Publications non specifically dedicated to the study of heat pipes 
Table 2 : Experimental database with the present FPHP

\begin{tabular}{|c|c|c|c|c|c|}
\hline$h_{\mathrm{v}}[\mathrm{mm}]$ & $\theta\left[{ }^{\circ}\right]$ & $f_{\mathrm{r}}$ & $Q[\mathrm{~W}]$ & $r_{\text {th }}[\mathrm{K} / \mathrm{W}]$ & $\Delta P_{\text {cap }} / \Delta x[\mathrm{~Pa} / \mathrm{m}]$ \\
\hline \multirow{2}{*}{$1 \mathrm{~mm}$} & \multirow{2}{*}{0} & \multirow{2}{*}{$18 \%$} & 28 & 0.21 & $175 \pm 20$ \\
\hline & & & 45 & 0.215 & $241 \pm 22$ \\
\hline \multirow{13}{*}{$2 \mathrm{~mm}$} & \multirow{7}{*}{0} & \multirow{3}{*}{$9 \%$} & 28 & 0.29 & $96 \pm 20$ \\
\hline & & & 45 & 0.24 & $137 \pm 23$ \\
\hline & & & 88 & 0.26 & $336 \pm 24$ \\
\hline & & \multirow{4}{*}{$13 \%$} & 28 & 0.27 & $62 \pm 16$ \\
\hline & & & 45 & 0.26 & $118 \pm 19$ \\
\hline & & & 57 & 0.25 & $186 \pm 9$ \\
\hline & & & 88 & 0.26 & $312 \pm 15$ \\
\hline & \multirow{3}{*}{2.5} & \multirow{3}{*}{$10 \%$} & 0 & - & $245 \pm 10$ \\
\hline & & & 44 & 0.26 & $407 \pm 26$ \\
\hline & & & 59 & 0.26 & $516 \pm 20$ \\
\hline & \multirow{3}{*}{5} & \multirow{3}{*}{$10 \%$} & 0 & - & $485 \pm 23$ \\
\hline & & & 28 & 0.26 & $617 \pm 52$ \\
\hline & & & 44 & 0.25 & $720 \pm 61$ \\
\hline
\end{tabular}


Table 3 : Experimental results of Rullière et al. (calculated from [4])

\begin{tabular}{|c|c|c|c|c|c|}
\hline $\boldsymbol{h}_{\mathrm{v}}[\mathrm{mm}]$ & $\boldsymbol{\theta}\left[{ }^{\circ}\right]$ & $\boldsymbol{f}_{\mathrm{r}}$ & $\boldsymbol{Q}[\mathbf{W}]$ & $\boldsymbol{r}_{\text {th }}[\mathbf{K} / \mathrm{W}]$ & $\Delta \boldsymbol{P}_{\text {cap }} / \Delta \boldsymbol{x}[\mathbf{P a} / \mathbf{m m}]$ \\
\hline \multirow{3}{*}{$1.6 \mathrm{~mm}$} & \multirow{3}{*}{0} & \multirow{3}{*}{$9.7 \%$} & 85.5 & 0.049 & 207 \\
\cline { 4 - 7 } & & & 119.7 & 0.047 & 327 \\
\cline { 4 - 6 } & & & 153.9 & 0.050 & 471 \\
\hline
\end{tabular}

